\title{
Inégalités d'accès aux ressources de la ville analysées à l'aide des mobilités quotidiennes
}

Approche méthodologique exploratoire à São Paulo

Inequalities in access to the resources of the city of São Paulo, analyzed in reference to residents' daily mobility practices

An exploratory methodological approach

\section{Desigualdad de acceso a los recursos urbanos analizada a} través de la movilidad cotidiana

\section{Enfoque metodológico exploratorio en la ciudad de San Pablo}

Florent Demoraes, Marie Piron, Silvana Zioni et Sylvain Souchaud

Volume 56, numéro 158, septembre 2012

Version originale soumise en mars 2012. Version révisée reçue en décembre 2012.

URI : https://id.erudit.org/iderudit/1014555ar

DOI : https://doi.org/10.7202/1014555ar

Aller au sommaire du numéro

Éditeur(s)

Département de géographie de l’Université Laval

ISSN

0007-9766 (imprimé)

1708-8968 (numérique)

Découvrir la revue

Citer cet article

Demoraes, F., Piron, M., Zioni, S. \& Souchaud, S. (2012). Inégalités d'accès aux ressources de la ville analysées à l'aide des mobilités quotidiennes : approche méthodologique exploratoire à São Paulo. Cahiers de géographie du Québec, 56(158), 463-490. https://doi.org/10.7202/1014555ar

\section{Résumé de l'article}

Cet article, avant tout méthodologique, explore la dispersion spatiale des lieux d'activité (étude et travail) fréquentés au quotidien par des individus au regard de leurs caractéristiques sociodémographiques et de leur lieu de résidence, dans l'agglomération de São Paulo. L'étude repose sur l'exploitation de données d'enquêtes sur les systèmes de mobilités réalisées auprès d'un échantillon de ménages. L'accent est mis sur la démarche qui fait intervenir une série d'analyses centrographiques que l'on combine dans un deuxième temps à une analyse typologique. Nous montrons l'intérêt de recourir à ces outils pour appréhender et visualiser les inégalités d'accès aux ressources urbaines en lien avec le cycle de vie des individus, la hiérarchie sociale et le lieu de résidence. 


\section{Inégalités d'accès aux ressources de la ville analysées à l'aide des mobilités quotidiennes Approche méthodologique exploratoire à São Paulo}

\author{
Inequalities in access to the resources of \\ the city of São Paulo, analyzed in reference \\ to residents' daily mobility practices. \\ An exploratory methodological approach \\ Desigualdad de acceso a los recursos \\ urbanos analizada a través de la movilidad \\ cotidiana. Enfoque metodológico \\ exploratorio en la ciudad de San Pablo
}

\author{
Florent DEMORAES \\ Laboratoire ESO-Rennes, UMR 6590 CNRS \\ Université de Rennes 2 \\ Florent.Demoraes@univ-rennes2.fr \\ Marie PIRON \\ IRD-UMR 8586 PRODIG \\ Centre de recherche d'Île-de-France \\ Marie.Piron@ird.fr \\ Silvana ZIONI \\ Universidade Federal do ABC - Brésil \\ Silvana.Zioni@ufabc.edu.br \\ Sylvain SOUCHAUD \\ IRD - URMIS \\ Université Paris Diderot \\ Sylvain.Souchaud@ird.fr
}

\section{Résumé}

Cet article, avant tout méthodologique, explore la dispersion spatiale des lieux d'activité (étude et travail) fréquentés au quotidien par des individus au regard de leurs caractéristiques sociodémographiques et de leur lieu de résidence, dans l'agglomération de São Paulo. L'étude repose sur l'exploitation de données d'enquêtes sur les systèmes de mobilités réalisées auprès d'un échantillon de ménages. L'accent est mis sur la démarche qui fait intervenir une série d'analyses centrographiques que l'on combine dans un deuxième temps à une analyse typologique. Nous montrons l'intérêt de recourir à ces outils pour appréhender et visualiser les inégalités d'accès aux ressources urbaines en lien avec le cycle de vie des individus, la hiérarchie sociale et le lieu de résidence.

\section{Mots-clés}

Inégalité d'accès, ressources urbaines, lieu de résidence, mobilité quotidienne, cycle de vie, hiérarchie sociale, découplage spatial, analyse centrographique, analyse typologique, São Paulo. 


\begin{abstract}
This article, primarily methodological in nature, examines the spatial distribution of places throughout São Paulo that people frequent on a daily basis for study and work, while taking into consideration their socio-demographic characteristics and place of residence. The study draws on surveys carried out among a sample of households, as a means to derive a better understanding of their mobility practices. Particular emphasis is placed on the methodological approach which is based on a series of centrographic analyses we combine with a typological analysis. We demonstrate the value of using these tools to pinpoint and reflect inequalities in access to urban resources and the relationship between those inequalities and the life cycle of the individuals involved, social hierarchy and their place of residence.
\end{abstract}

\title{
Keywords
}

Inequality of access, urban resources, place of residence, daily mobility, life cycle, social hierarchy, spatial mismatch, centrographic analysis, typological analysis, São Paulo.

\section{Resumen}

Este artículo, ante todo metodológico, explora la dispersión espacial de los sitios de actividades cotidianas (estudio y trabajo) frecuentados por los individuos, según sus características socio-demográficas y el lugar de residencia, en la aglomeración de San Pablo. El estudio se apoya en el tratamiento de datos de encuestas sobre sistemas de movilidad realizadas en una muestra de hogares. Se hace hincapié en la metodología que implementa una serie de análisis centrográficos enriquecidos en un segundo tiempo por un análisis tipológico. Se demuestra el interés de tales herramientas para visualizar y comprender las desigualdades de acceso a los recursos urbanos relacionados con el ciclo de vida de los individuos, con la jerarquía social y con el lugar de residencia.

\section{Palabras claves}

Desigualdad de acceso, recursos urbanos, lugar de residencia, movilidad cuotidiana, ciclo de vida, jerarquía social, desfase espacial, análisis centro gráfico, análisis tipológico, San Pablo.

\section{Introduction}

Tout le monde n’accède pas à la ville de la même façon. Tout le monde ne fréquente pas les mêmes lieux. Ces différences s'observent quelle que soit la ville étudiée et s'expliquent par un très grand nombre de facteurs étroitement imbriqués entre eux. De façon schématique, on recense i) des facteurs liés à l'individu et à son entourage, ii) des facteurs liés au moyen de transport, et iii) des facteurs liés à la ressource urbaine (emploi, éducation, loisirs, santé, etc.).

En Amérique latine, la question des inégalités d'accès aux ressources urbaines se pose avec une grande acuité, du fait des caractéristiques de l'urbanisation sur ce continent (Demoraes et al., 2010) : des métropoles millionnaires très étalées et peu denses, marquées par de profondes inégalités sociales (Janoschka, 2002 ; Borsdorf, 2003), une très forte ségrégation sociorésidentielle (Clichevsky, 2000; Lungo et Baires, 2001 ; Rodríguez et Arriagada, 2004), une inéquitable répartition des emplois dans 
la ville et des systèmes de transport en commun pas toujours adaptés à la demande (Vasconcellos, 1996; Montezuma, 2003 ; Figueroa, 2005). Les métropoles d’Amérique latine suivent ainsi un modèle d'urbanisation relativement convergent qui exerce des contraintes fortes sur les mobilités résidentielles et quotidiennes de leurs habitants (Dureau, 2006).

São Paulo n’échappe pas à ce schéma et est, elle aussi, caractérisée par une forte inégalité d'accès aux ressources urbaines, comme l'indiquent notamment Vasconcellos (2005), Kilroy (2007) ou Villaça (2007). Si les grandes enquêtes origine-destination réalisées par la Companhia do metrô ${ }^{1}$ font ressortir, sur l'ensemble de l'agglomération, des contrastes en termes de mobilité quotidienne, en fonction des revenus, de l'âge, du sexe, du mode de transport et du motif de déplacement, d'autres variables pouvant influencer les déplacements ne sont pas recueillies lors de ces enquêtes. Il s'agit notamment d'indicateurs qui permettent de définir l'étape à laquelle un individu se trouve dans son cycle de vie. Par ailleurs, l'exploitation des grandes enquêtes origine-destination reste somme toute limitée, dans la mesure où il n'est pas possible de récupérer les données individuelles produites dans le cadre de ces enquêtes. Seules sont disponibles des données agrégées, avec des combinaisons de variables prédéfinies et déjà partitionnées en classes.

Les enquêtes réalisées par notre équipe dans le cadre du programme de recherche METAL $^{2}$ nous permettent d'explorer plus finement les écarts enregistrés dans l'accès aux ressources urbaines à partir d'une dizaine de zones de résidence, déclinés selon une série de variables individuelles. Nos enquêtes permettent également de répondre aux questions suivantes: comment les espaces fréquentés au quotidien se différencient-ils selon le lieu de résidence et les caractéristiques sociodémographiques des individus? Quels sont les lieux de résidence où les disparités d'accès à la ville sont les plus marquées? Quels sont les espaces d'activité associés aux différentes étapes du cycle de vie des individus? Quels enseignements peut-on en tirer pour évaluer le niveau d'adéquation spatiale entre lieu de résidence et lieu de travail?

Dans une première partie, nous détaillons le terrain d'étude, le corpus de données, les outils mobilisés et la méthode élaborée pour répondre à ces questions. Dans la seconde partie, nous analysons la dispersion des espaces fréquentés par les individus différenciés selon deux caractéristiques (motif de déplacement, revenus) fréquemment retenues pour mettre en évidence des disparités en termes d'espaces pratiqués au quotidien. Puis nous proposons de replacer les individus de façon plus globale dans leur cycle de vie et dans la hiérarchie sociale afin d'en explorer l'influence sur les destinations.

1 La Companhía do metrô est l'organisme qui assure l'exploitation du métro à São Paulo. Les pouvoirs publics lui confient également la réalisation de grandes enquêtes origine-destination auprès des ménages tous les 10 ans. Entre deux enquêtes, sont réalisées des projections à partir d'un échantillon plus restreint. Les dernières enquêtes datent de 1997 et 2007. En 2002, des projections ont été réalisées à partir de l'enquête de 1997.

2 Le programme METAL (Métropoles d'Amérique latine dans la mondialisation: reconfigurations territoriales, mobilité spatiale, action publique) est dirigé par Françoise Dureau. Il s'inscrit dans le cadre de l'ANR/AIRD «Les Suds aujourd'hui» et porte sur trois métropoles d'Amérique latine, Bogotá, Santiago du Chili et São Paulo. Pour en savoir plus: http://www.mshs.univ-poitiers.fr/migrinter/ index.php?text=institutionnel/METAL\&lang=fr. 


\section{Terrain d'étude, corpus de données et méthode}

\section{Présentation de l'agglomération de São Paulo}

L'agglomération de São Paulo rassemblait 19,7 millions d'habitants en 2010 (Instituto Brasileiro de Geografia e Estatísticas [IBGE]) répartis sur près de 1660 kilomètres carrés $^{3}$. Même si, globalement, la disponibilité des emplois formels semble s'être accrue $^{4}$, les activités productives restent inéquitablement réparties dans l'espace métropolitain. Malgré un redéploiement de ces activités amorcé dès 2000, on trouve les emplois encore aujourd'hui principalement dans l'espace central (figure 1), le secteur de l'ABC ${ }^{5}$ et aux abords de l'aéroport international de Guarulhos, au nordest. L'informalité reste cependant très présente et les écarts de revenus extrêmement marqués. En 2009, le salaire minimum était de l'ordre de 465 reais, le salaire médian avoisinait 1000 reais et le salaire moyen était de 1832 reais, soit respectivement 177 , 381 et 698 euros. L'offre éducative est quant à elle distribuée de façon plus homogène, notamment pour l'éducation primaire et secondaire. Pour les universités, il faut distinguer le public et le privé. Le public est concentré dans quelques lieux ${ }^{6}$. Le privé représente une offre abondante et est comparativement un peu mieux réparti dans l'ensemble de l'agglomération.

Pendant la période 1997-2007, le parc automobile a connu une croissance de 16\% (Cia do Metrô, 2008), ce qui implique une proportion aujourd'hui plus élevée de déplacements motorisés individuels, avec près d'un quart des trajets réalisés en voiture. Les transports publics collectifs comptent pour 55\% des 25 millions de déplacements motorisés quotidiens. La part des déplacements à pied est loin d'être négligeable, puisque ces derniers s'élèvent à 12 millions par jour (près d'un tiers des déplacements quotidiens). Par ailleurs, la ville centre est dotée d'un métro circulant en 2011 sur un réseau de 61,3 km et comportant 55 stations, ce qui est peu au regard de l'étendue de l'agglomération (figure 1) et ce qui accentue les inégalités d'accès à la ville (Villaça et Zioni, 2005).

\section{Les sources de données mobilisées}

Les enquêtes par questionnaire sur les systèmes de mobilité ont été menées à São Paulo par l'équipe du programme METAL entre juin et septembre 2009 dans 11 zones d'étude

3 D'est en ouest, la partie urbanisée de São Paulo, dans sa plus grande dimension, s'étale sur près de 90 $\mathrm{km}$, et sur près de $60 \mathrm{~km}$ du nord au sud.

4 Entre les recensements de 2000 et de 2010, les emplois formels ont augmenté de 30\%, chiffre supérieur à l'augmentation de la population, évaluée à 10,1\% (IBGE, 2010).

5 Le secteur $\mathrm{ABC}$ est un grand bassin industriel (secteur automobile, usines agroalimentaires, pétrochimie...) situé au sud-est de São Paulo. Ce secteur englobe, entre autres, trois importants municipes: Santo André, São Bernardo do Campo, São Caetano do Sul. La zone 10 incluse dans l'enquête de l'équipe METAL se situe dans ce secteur (figure 1).

6 Le public, soumis à une forte sélection à l'entrée est regroupé notamment dans les secteurs Vila Clementino (au sud du centre historique, voir figure 1) et Cidade Universitaria (à la limite extérieure ouest de l'espace central). 
(8 à l'intérieur du municipe de São Paulo et 3 dans sa périphérie métropolitaine) ${ }^{7}$. Cette série d'enquêtes, dont une partie est analysée dans cet article, permet d'appréhender les mobilités quotidiennes, résidentielles et migratoires de chaque individu.

La sélection des zones d'enquête a été guidée par plusieurs critères:

- la dynamique démographique et la composition sociale de la population, évaluées à travers les projections de population à l'année 2009, calculées par l'IBGE à partir du dernier recensement disponible ${ }^{8}$;

- la localisation au sein du système métropolitain (espace central, péricentre, périphérie) ;

- les caractéristiques du marché du logement, le potentiel de transformation du bâti et l'intervention urbanistique (opérations de requalification urbaine, grands projets urbains) évalués dans les documents cadres de planification et à partir de la connaissance qu'a notre équipe de ces thématiques, localement.

Les zones d'étude composent ainsi une mosaïque «illustrative» (et non représentative au sens statistique du terme) des profils socioéconomiques, des conditions de logement et des processus d'urbanisation de l'agglomération de São Paulo (figure 1 et tableau 1). Elles nous permettent de définir une grande variété de stratégies de mobilité quotidienne pour accéder aux ressources de la ville.

Dans l'ensemble de São Paulo et de sa périphérie, notre enquête a été réalisée auprès de 907 ménages, soit 2956 individus, et 55 ont fait l'objet d'entretiens approfondis ${ }^{9}$. Les ménages ont été sélectionnés dans chaque zone selon un plan de sondage aréolaire à deux degrés (îlots, ménages), en suivant la méthode mise au point par Dureau et al. (1989). Cette méthode permet de garantir la représentativité des ménages pour chaque zone d'enquête ${ }^{10}$. L'échantillon extrapolé représente 140019 individus, ce qui correspond à la population totale des neuf zones où l'enquête a été menée.

Pour étudier les pratiques de mobilité quotidienne dans leur dimension spatiale, une base de données a été mise en place dans un système d'information géographique (SIG). Elle rassemble un ensemble structuré et documenté de couches thématiques, notamment les réseaux de métro et de train, l'occupation du sol, les secteurs censitaires (21 744), le découpage en áreas de ponderação (812) ${ }^{11}$, districts (162) et municipalités (39) ainsi que les limites des zones de l'enquête.

7 Sur les onze zones initiales, nous ne présentons les résultats que de neuf d'entre elles. En effet, une première zone a dû être écartée car les enquêteurs n’ont pu entrer en contact avec les personnes sélectionnées (lotissements fermés). Une deuxième zone a également été exclue de notre analyse car nous ne pouvions extrapoler les valeurs recueillies (erreur au niveau du plan de sondage).

8 Le dernier recensement disponible au moment de réaliser les enquêtes datait de 2000.

9 L’exploitation des entretiens n’est pas développée dans cet article.

10 Les individus sont donc représentatifs uniquement de leur zone de résidence, pas de l'ensemble de la ville.

11 Il s'agit d'un regroupement de secteurs censitaires. Cet échelon permet la restitution des résultats sous forme de cartes sur l'ensemble de la Région métropolitaine de São Paulo. 

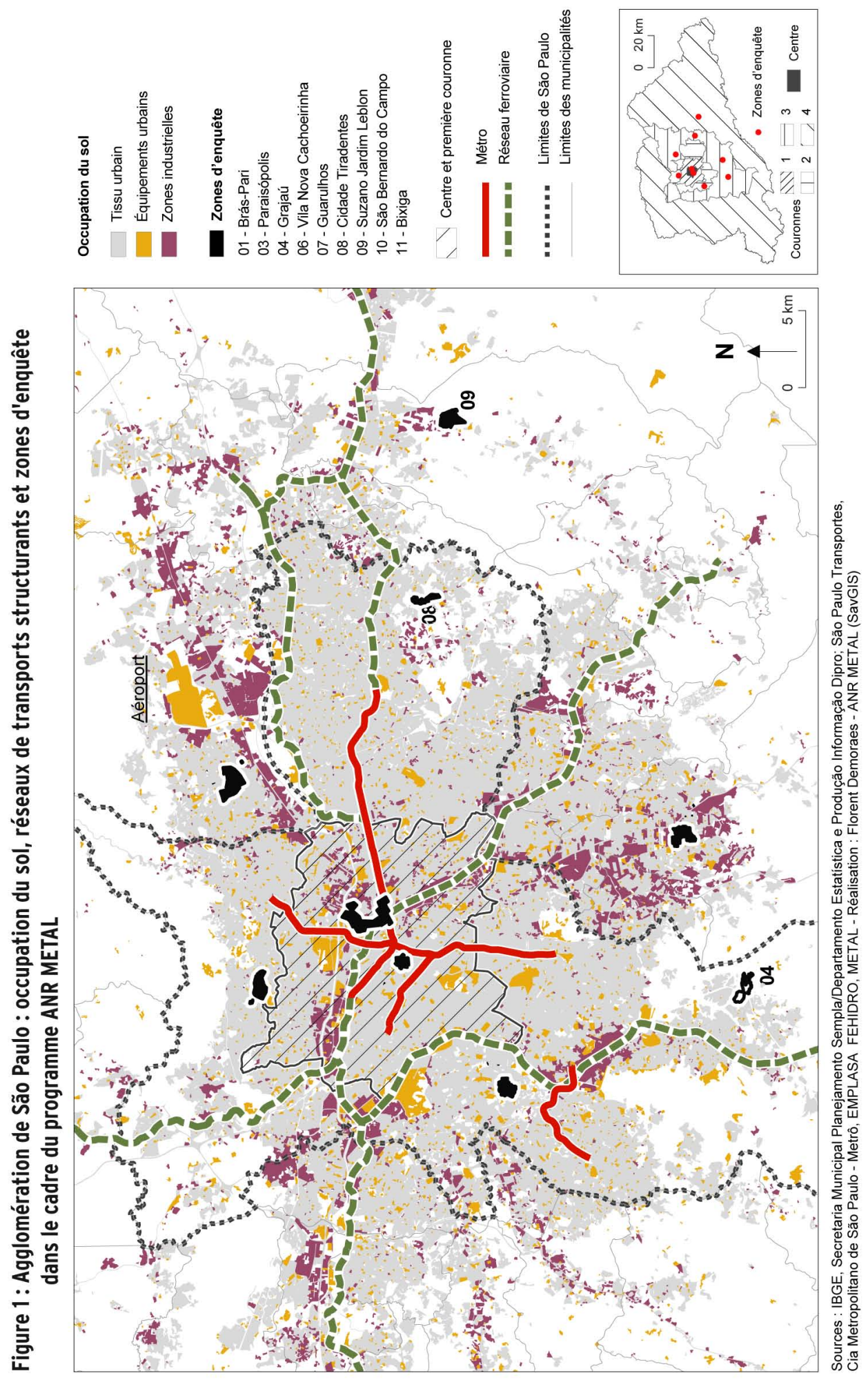


\section{Tableau 1 Caractérisation des zones d'enquête à São Paulo dans le cadre du programme ANR METAL}

\begin{tabular}{|c|c|}
\hline Zones METAL & Description \\
\hline 01 - Brás/Pari & $\begin{array}{l}\text { Forte concentration d'emplois industriels, d'activités commerciales et de services, } \\
\text { Brás/Pari est l'un des principaux pôles d'attraction de la métropole, et maintient } \\
\text { ainsi sa condition de centre, consolidé il y a plus d'un siècle. Cette zone se } \\
\text { caractérise aussi par son caractère résidentiel populaire et par une bonne } \\
\text { accessibilité, dans la mesure où elle est desservie par plusieurs systèmes de } \\
\text { transports publics. }\end{array}$ \\
\hline 03 - Paraisópolis & $\begin{array}{l}\text { Favela, enclave de ménages à revenus modestes située à l'intérieur d'un secteur } \\
\text { résidentiel à hauts revenus et qualifié de prestigieux. Paraisópolis présente } \\
\text { localement des caractéristiques morphologiques et un équipement réduit en } \\
\text { infrastructures routières qui y limitent la circulation. En revanche, elle bénéficie } \\
\text { d'une position assez centrale dans l'agglomération et se trouve à proximité de } \\
\text { plusieurs grands axes routiers qui la relient assez facilement au centre. }\end{array}$ \\
\hline 04 - Grajaú & $\begin{array}{l}\text { Zone d'occupation irrégulière et de logements précaires située au sein d'un } \\
\text { espace de protection naturelle, essentiellement résidentielle et composée de } \\
\text { ménages à faibles revenus. On y trouve aussi à proximité des ménages à hauts } \\
\text { revenus. La dotation en infrastructures viaires et la dessserte en transports en } \\
\text { commun y sont limitées. }\end{array}$ \\
\hline $\begin{array}{l}06 \text { - Vila Nova } \\
\text { Cachoeirinha }\end{array}$ & $\begin{array}{l}\text { Zone populaire résidentielle formée à partir de lotissements implantés sur des } \\
\text { terrains vallonnés et segmentés par des ruisseaux qui conditionnent son réseau } \\
\text { viaire. Cette zone consolidée, où vivent des ménages à revenus modestes et } \\
\text { moyens, bénéficie d'une relative proximité aux secteurs centraux de SP. }\end{array}$ \\
\hline 07 - Guarulhos & $\begin{array}{l}\text { Située dans l'espace central du municipe de Garulhos, cette zone est caractérisée } \\
\text { par un usage résidentiel de classe moyenne, et dispose d'une accessibilité } \\
\text { favorisée par la proximité du bassin d'emplois autour de l'aéroport. }\end{array}$ \\
\hline $\begin{array}{l}08 \text { - Cidade } \\
\text { Tiradentes }\end{array}$ & $\begin{array}{l}\text { Logements sociaux organisés en grands ensembles construits par les pouvoirs } \\
\text { publics en marge desquels se trouve une favela. Son éloignement des centres } \\
\text { d'emplois implique un volume élévé de mouvements pendulaires. Elle se } \\
\text { caractérise également par une desserte peu performante en transports en } \\
\text { commun. }\end{array}$ \\
\hline $\begin{array}{l}09 \text { - Suzano Jardim } \\
\text { Leblon }\end{array}$ & $\begin{array}{l}\text { Zone à l'extrême est de la Région métropolitaine de São Paulo, très éloignée des } \\
\text { centres d'emplois, assez mal connectée au reste de la RMSP. Se caractérise par } \\
\text { une faible densité résidentielle et par la présence de demeures entourées de } \\
\text { vastes jardins, occupées par des populations à revenus moyens ou faibles. }\end{array}$ \\
\hline $\begin{array}{l}10 \text { - São Bernardo } \\
\text { do Campo }\end{array}$ & $\begin{array}{l}\text { Localisée entre les deux grandes zones industrielles implantées le long d'axes } \\
\text { routiers structurants de la RMSP, cette zone occupée par des ménages à revenus } \\
\text { moyens ressort comme un îlot résidentiel implanté au milieu d'usines. Locale- } \\
\text { ment, le réseau routier est peu hiérarchisé et peu de grands axes innervent la } \\
\text { zone rendant la circulation difficile à l'intérieur de cette dernière. Les stratégies } \\
\text { de mobilité dépendent essentiellement de l'automobile. }\end{array}$ \\
\hline 11 - Bixiga & $\begin{array}{l}\text { Zone résidentielle populaire implantée sur des terrains à topographie accidentée } \\
\text { qui façonne son réseau viaire et la forme de ses îlots. Est délimitée par le centre } \\
\text { historique et l'Avenue Paulista, deux pôles d'emplois et d’activités. }\end{array}$ \\
\hline
\end{tabular}




\section{Méthode: analyses centrographique et typologique}

\section{Caractérisation et comparaison de semis de points: I'analyse centrographique}

La première étape a consisté à localiser les lieux fréquentés au quotidien par les individus pour le travail et les activités scolaires (études primaires, secondaires et supérieures ${ }^{12}$. Nous excluons de facto ici les individus exerçant une activité à leur lieu de résidence, qui représentent 7022 personnes sur les 61045 actifs découlant d'une extrapolation à partir de notre échantillon, soit près de $12 \%$ du total. Nous excluons aussi les 5496 (9\%) personnes exerçant une profession difficile à localiser (vendeur ambulant, travailleur de rue, travailleur se déplaçant dans un véhicule).

Les lieux fréquentés au quotidien ont été positionnés dans un premier temps sur le centroïde des districts de destination, à défaut d'une localisation plus fine. En effet, les adresses de destination n'ont pas pu être recueillies avec précision lors des enquêtes, compte tenu des problèmes de nomenclature qui peuvent exister dans une agglomération de cette envergure. Il est important de préciser aussi que nous n’avons pas pu associer tous les lieux de destination à des districts, du fait de problèmes liés au recueil même ou au codage ultérieur. Cette limite concerne surtout les navettes domicile-travail et explique les différences entre l'effectif de l'échantillon initial et celui de l'échantillon localisé (les effectifs sont annotés dans les figures 2, 3 et 6).

Cette localisation par centroïde restait parfois peu précise, car certains districts en périphérie sont très vastes et partiellement urbanisés (certains pouvant atteindre près de 52000 ha). Ce manque de précision est malgré tout à relativiser, car les districts périphériques étendus ne correspondent qu'à une très faible proportion des lieux de destination. En effet, au sein de notre population d'enquête, $68 \%$ des déplacements pour le travail et $62 \%$ des déplacements pour les activités scolaires s'effectuent vers des districts dont la superficie est inférieure à 1350 ha (surface médiane des districts). Ainsi, pour les deux tiers des déplacements, le choix du centroïde du district de destination est acceptable pour localiser les lieux fréquentés au quotidien, d'autant que notre échelle de restitution ici est celle de l'agglomération.

Dans le cas particulier des districts périphériques, le centre géométrique naturel est parfois localisé dans des secteurs inhabités, faussant de facto le calcul de la dispersion des lieux fréquentés. Nous avons corrigé ce problème en replaçant les centroïdes sur les parties urbanisées des districts ${ }^{13}$. Le positionnement de l'ensemble des lieux de destination a été in fine vérifié afin d'éviter les espaces boisés et les étendues d'eau.

Ensuite, dans le SIG, nous appliquons sur ces lieux une analyse centrographique, méthode introduite par Bachi en 1963, afin de calculer des indicateurs synthétiques

12 Ces trajets représentent près de 40\% des trajets quotidiens (Cia do Metrô, 2008). Les 60\% restants englobent tous les autres motifs, notamment les retours au domicile.

13 Pour ce faire, nous avons calculé le barycentre pondéré par la population disponible à une échelle inférieure, les secteurs censitaires. Ces derniers s'emboîtent parfaitement dans les districts (découpage hiérarchique). 
de dispersion spatiale (calcul des points moyens ${ }^{14}$, des distances types et des ellipses de dispersion). Les calculs du centre moyen et de la distance type (influençant la taille de l'ellipse) sont ici pondérés par le nombre d'individus sur leur lieu de destination ${ }^{15}$.

L’analyse centrographique est particulièrement bien adaptée pour résumer et caractériser des semis de points à l'aide d'indicateurs (position moyenne, taille, forme, orientation). Elle permet également de visualiser et comparer l'agencement des ellipses les unes par rapport aux autres sur un espace donné.

Cette méthode a été abondamment appliquée à la thématique des mobilités quotidiennes (lieux de destination ou trajectoires), et d'autant plus ces 30 dernières années que la disponibilité de données localisées à l'adresse s'est accrue et que des outils permettant de mettre en œuvre cette méthode ont été intégrés dans les logiciels de SIG ${ }^{16}$. Donald et Goodchild (1983) l'ont par exemple appliquée à 1500 personnes dans la ville d'Halifax pour décrire, à différents moments d'une journée, les positions moyennes ainsi que les degrés de concentration spatiale et de ségrégation de différents sous-groupes de personnes, répartis suivant des caractéristiques sociodémographiques (classes d'âge, statut matrimonial, statut d'occupation du logement, langue parlée au sein du ménage, niveaux d'éducation, taux de motorisation). Noël et al. (2001) ont utilisé cette méthode pour établir les déterminants des stratégies de déplacement et les espaces d'action (ensemble de lieux fréquentés) de 200 cyclistes dans la région de Québec, qu'ils ont ensuite confrontés à l'organisation du territoire et au milieu de résidence. Morency (2006) l'emploie sur les données de l'enquête origine-destination de 1988 à l'échelle de l'agglomération de Montréal (échantillon de 65000 ménages) et analyse la distribution spatiale des segments de population différenciés selon la taille des ménages, les revenus, l'âge, le taux de motorisation et l'usage des transports en commun. Lord et al. (2009) recourent à cette méthode pour mesurer l'évolution, entre 1999 et 2006, des espaces d'action d'une centaine de personnes âgées résidant dans la banlieue de Québec dont ils dressent une typologie qu'ils interprètent au regard de la relocalisation des services de proximité et de critères sociodémographiques tels que l'âge, le niveau d'autonomie et les revenus. Imbert et al. (2009) ont testé cette méthode dans le cadre d'une enquête portant sur un peu plus de 900 individus, réalisée à Poitiers en 2005 et 2006. L'objectif était de décrire les mobilités qu'ils ont mises en balance avec le fonctionnement des ménages, les choix résidentiels, la localisation et les caractéristiques des résidences. Ce rapide aperçu des cas d'utilisation de l'analyse centrographique nous renseigne également sur les caractéristiques sociodémographiques employées pour appréhender les facteurs qui influencent les pratiques de mobilité quotidienne (voir section suivante).

14 Nous avons également calculé les points médians (davantage adaptés aux semis de points présentant différents agrégats). Les écarts de positionnement entre points moyens et points médians sont plus marqués pour les zones d'enquête les plus périphériques (destinations plus variées, trajets plus longs), mais n'excèdent pas $3,2 \mathrm{~km}$, ce qui est peu au regard de l'étendue de l'agglomération. Par ailleurs, ces écarts jouent uniquement sur le positionnement des ellipses, mais pas sur leur taille, leur orientation et leur forme. L'agencement des ellipses les unes par rapport aux autres ne change donc pas, quelle que soit la méthode retenue pour le calcul de leur centre.

15 L'outil retenu pour calculer les ellipses de dispersion est le SIG gratuit SavGIS (www.savgis.org) (voir Souris et al., 2007).

16 Marius Thériault, chercheur au Centre de recherche en aménagement et développement (CRAD) rattaché à l'Université Laval (Québec) a mis au point, en 1994, une application exécutable sur le logiciel Mapinfo (Mapstat). Cet outil a contribué à répandre l'utilisation de l'analyse centrographique parmi les géographes et aménageurs. 
Dans notre cas, cette méthode est d'autant plus simple à mettre en œuvre que nous ne considérons qu'une seule destination par jour et par individu. Le semis de points des destinations ne fluctue donc pas au cours d'une journée. Ceci étant, il est important de rappeler que pour calculer une ellipse, il faut au moins deux points (ici deux destinations différentes), condition qui n'est pas toujours remplie (voir la section des résultats). En effet, il arrive que tous les déplacements effectués au départ d'une zone n'aient qu'une seule destination. Ce cas s'observe notamment sur la figure 2 pour les déplacements vers un établissement scolaire, au départ de la zone 9. Cette dernière est située dans un district particulièrement vaste ( 9800 ha) et les individus scolarisés restent dans leur district de résidence.

De même, pour calculer une ellipse, il faut un effectif suffisant, sans quoi l'ellipse aura peu de sens (limite déjà évoquée par Imbert et al., 2009). Sur les figures 2, 3 et 6 , les effectifs sont variables selon les zones d'enquête et le critère de différenciation retenu (motif de déplacement, classe de revenus, classe sociodémographique). Ceci s'explique par le mode de sélection des zones d'enquête. Dans notre étude, nous avons tracé les ellipses uniquement lorsque le nombre de personnes appartenant à une classe sociodémographique et une zone d'enquête données est au moins égal à cinq ${ }^{17}$. Enfin, il arrive qu'aucun individu appartenant à une classe sociodémographique donnée ne réside dans certaines zones d'enquête ${ }^{18}$.

Ces différences d'effectifs ne constituent pas pour autant un obstacle à la comparaison des ellipses entre elles. En effet, la taille des ellipses n'est pas fonction de l'effectif. Sur les figures 3 et 6 , les coefficients de corrélation $R$ entre effectifs soumis à l'enquête et surfaces elliptiques valent respectivement 0,13 et $0,11^{19}$. L'absence d'ellipse renseigne également sur les particularités des pratiques de déplacement dans une zone donnée.

\section{Choix des variables: exploration préliminaire à l'aide de l'approche univariée}

De nombreuses études mettent en évidence le lien entre variables individuelles (sexe, âge, activité, etc.), variables relatives aux ménages (revenus du ménage, conditions de logement, etc.), variables contextuelles (localisation de la parentèle, caractéristiques du quartier, etc.) et pratiques de mobilité quotidienne. Outre les références mentionnées dans le paragraphe précédent, on peut faire allusion aux travaux de Baccaïni. Cette auteure montre qu'en Île-de-France, «les trajets domicile-travail varient fortement selon les professions... D’autres caractéristiques des actifs ont un effet sur leur mobilité quotidienne: en particulier, la propension des actifs à faire une longue navette varie selon leur âge ou leur choix résidentiel, à catégorie socioprofessionnelle donnée» (1996: 109). Dans un contexte sud-américain, Delaunay (2010) mesure, à partir des données de recensement, l'évolution des mobilités quotidiennes déclinées selon la catégorie socioprofessionnelle et le cycle de vie dans l'agglomération de Santiago du Chili.

17 On observe deux cas seulement en dessous de ce seuil sur la figure 6. Ce seuil a été choisi compte tenu de l'effectif par classe sur notre population enquêtée. Sur la figure 6, les individus ont été répartis en cinq classes sociodémographiques. Certaines catégories d'individus sont peu présentes localement. C'est donc sur cette figure que l'effectif est le plus faible (en moyenne 29 individus par classe et par zone, avec un maximum de 63).

18 Trois cas se présentent sur la figure 6.

19 Sur la figure 2, ce coefficient est légèrement plus élevé $(0,41)$. Cela s'explique par le fait que les déplacements effectués pour le travail ont des destinations plus variables que pour les études (logique de proximité). Ainsi, lorsque l'effectif augmente, la variabilité des destinations augmente aussi, pour le travail plus que dans le cas des trajets pour étude. 
Figure 2 : Dispersion des lieux de destination des individus selon le motif de déplacement et leur lieu de résidence
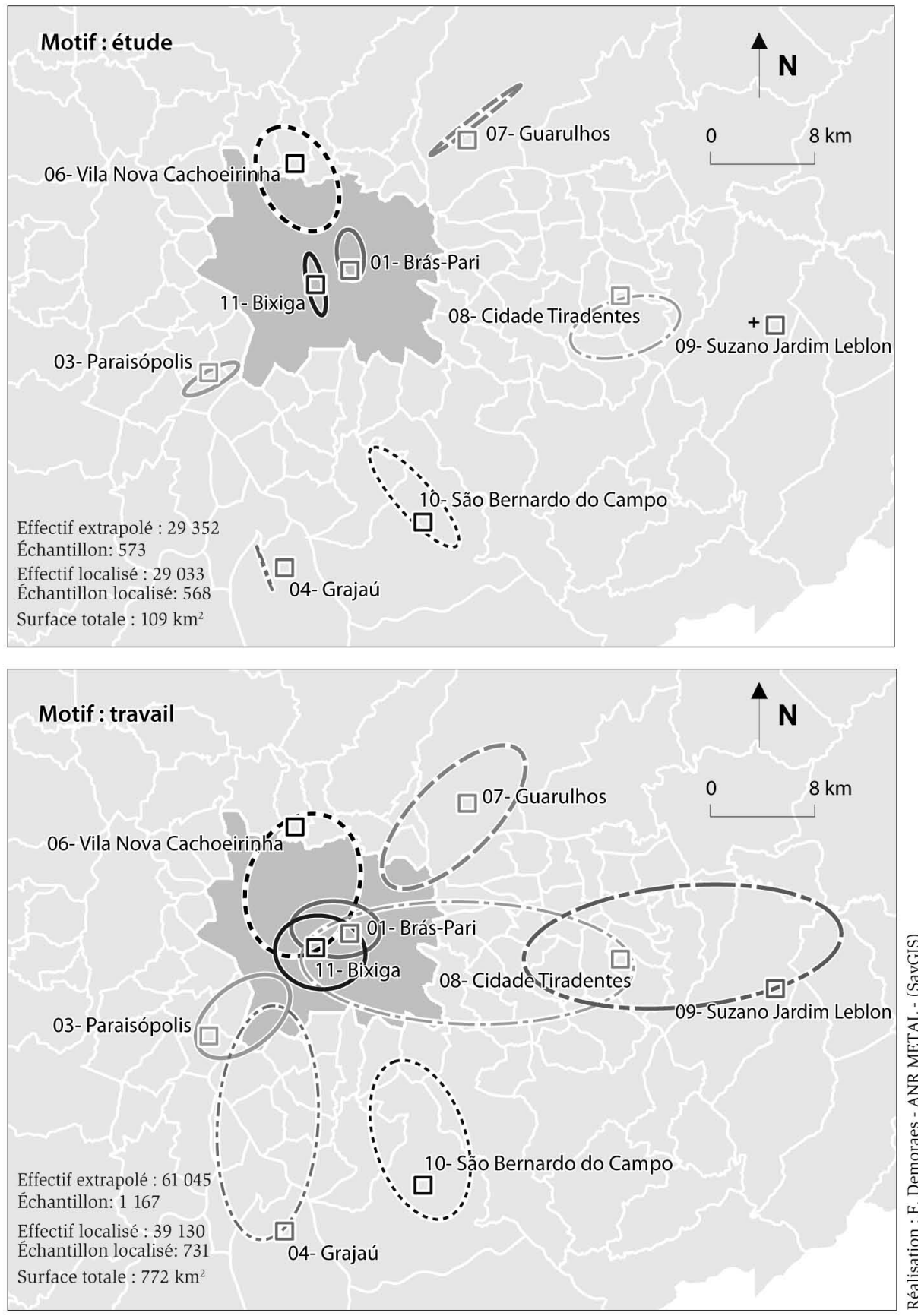

Limite extérieure de la première couronne

Les divisions à l'extérieur de la première couronne représentent les districts

Cas particuliers (absence d'ellipse) : + district de destination = district de résidence 
D’autres auteurs analysent la façon dont interagissent les dimensions quotidienne et résidentielle de la mobilité, très longtemps étudiées séparément comme le rappelle, entre autres, Lévy (2009) ${ }^{20}$. Si ces études sont de plus en plus fréquentes à l'échelle des agglomérations urbaines en Europe et Amérique du Nord, rares sont celles qui portent sur des villes latino-américaines. Berger et Beaucire (2002) et Berger (2004) décryptent l'articulation entre les choix résidentiels, la localisation des emplois et la durée des navettes pour les périurbains de Paris. Pochet et Routhier (2002) étudient l'impact des migrations résidentielles sur l'allongement des distances domicile-travail dans la grande région urbaine lyonnaise. Rodríguez (2007) analyse les interactions entre mobilités quotidiennes et résidentielles et ségrégation résidentielle socioéconomique à Santiago du Chili. Dureau et al. (2012) analysent les inégalités d'accès au reste de la ville chez les résidants d'un quartier de l'ouest de Bogotá, inégalités remises en perspective avec la trajectoire biographique des individus et la transformation de leur quartier sur une décennie. Ces analyses permettent de contribuer à la réflexion sur le concept de "mobilités spatiales» (Lévy et Dureau, 2002), entendu comme l'ensemble des mobilités expérimentées par les individus et leur famille à différents rythmes et échelles.

Dans notre étude, la première étape a consisté à choisir les variables parmi celles employées habituellement, compte tenu de l'influence qu'elles peuvent avoir sur les mobilités quotidiennes. De nombreux auteurs (Ântico, 2005; Aranha, 2005), exploitant les données des grandes enquêtes origine-destination réalisées à São Paulo par la Companhia do metrô, soulignent que les espaces de mobilité sont fortement dépendants des motifs de déplacement et du revenu des ménages et que cette tendance n’a que peu évolué ces 15 dernières années. Demoraes et al. (2010) indiquent que 49,6\% des déplacements pour le travail sont effectués, en 2007 à São Paulo, à l'intérieur de la zone de résidence des individus, alors que le chiffre atteint 85,6\% pour les déplacements pour étude. Comme dans la plupart des grandes villes, les individus à São Paulo parcourent donc des distances plus longues pour accéder à un emploi que pour se rendre à leur lieu d'étude. De son côté, Vasconcellos (2005) montre que le pourcentage d'immobiles va décroissant avec l'augmentation des revenus alors que les distances moyennes parcourues vont croissant. L'accès à la mobilité, à São Paulo, semble donc être directement corrélé aux revenus.

Les données recueillies lors de l'enquête METAL permettent-elles de confirmer ces constats ou leur apportent-elles des nuances?

Pour répondre à cette première interrogation, nous explorons, à l'aide d'ellipses, la distribution spatiale des lieux pratiqués au quotidien suivant le motif du déplacement (étude/travail) et les revenus ${ }^{21}$, différenciée selon le lieu de résidence (figures 2 et 3 ).

\section{Espaces de mobilité, cycle de vie et hiérarchie sociale: approche multivariée}

Le principe, ensuite, est d'explorer de façon plus approfondie l'influence des caractéristiques sociodémographiques, non plus prises une à une mais combinées entre elles, sur la dispersion des espaces fréquentés (position, taille, forme, orientation).

20 L'interaction des différents registres de la mobilité est un thème scientifique au cœur des débats actuels. Le sujet a notamment fait l'objet du $9^{\mathrm{e}}$ colloque Mobilités spatiales et fluidité sociale, de l'Association internationale des sociologues de langue française (AISLF) à Luxembourg, en mars 2009.

21 Tous les membres d'un ménage ont le même revenu. 
En effet, les variables sociodémographiques sont en partie liées et varient de façon plus ou moins concomitante tout au long du cycle de vie. En règle générale, de façon schématique, un individu au cours de sa vie passera progressivement de l'école au monde du travail, améliorera ses revenus et accédera à la propriété résidentielle.

L'une des originalités de l'enquête METAL est justement de décrire, pour chaque individu, à la fois ses mobilités quotidiennes et ses caractéristiques sociodémographiques telles que l'âge, le statut d'occupation du logement (propriétaire/locataire) ou encore l'ancienneté dans le logement. Ces caractéristiques donnent des indications sur l'étape à laquelle l'individu se trouve dans son cycle de vie que l'on peut confronter à son degré d'accès à la ville, ce qui constitue une source de données unique en son genre à São Paulo.

L'objectif est donc de définir une typologie des individus suivant la hiérarchie sociale et l'étape à laquelle ils se trouvent dans leur cycle de vie, et de comparer la dispersion des lieux de destination des individus regroupés par types, pour chacune des zones de résidence. Nous appliquons, par conséquent, une analyse des correspondances multiples (ACM) suivie d'une classification ascendante hiérarchique (CAH) à l'ensemble des personnes enquêtées, décrites selon les caractéristiques sociodémographiques suivantes: le sexe, l'âge, l'activité (étude ou travail), l'ancienneté de l'individu dans le logement, le statut d'occupation du logement (propriétaire, locataire, etc.), le niveau d'éducation ainsi que le revenu moyen du ménage auquel l'individu appartient (tableau 2). Cet enchaînement de méthodes constitue une analyse typologique et s'applique bien à des données d'enquêtes sur les systèmes de mobilité ${ }^{22}$. Cette analyse permet ainsi de situer les individus dans leur cycle de vie ${ }^{23}$ et dans la hiérarchie sociale.

La typologie est enrichie et illustrée par les indicateurs de déplacement mis en éléments supplémentaires ${ }^{24}$ : le mode de transport, le temps de trajet vers le lieu d'activité (travail ou étude), la destination (centre, première couronne, deuxième couronne, etc.) et un dernier indicateur qui permet d'estimer la distance parcourue vers le lieu de travail ou d'étude (exprimé en termes de voisinage calculé au moyen d'un graphe de contiguïté: sur le lieu de résidence, à l'intérieur du district de résidence, dans un district adjacent, dans un district non adjacent). Ces indicateurs viennent également complémenter et expliquer les écarts observés dans la dispersion des espaces fréquentés.

Les données et les méthodes ayant été présentées, l'objet de la deuxième partie est d'exposer les premiers résultats.

22 «L'analyse des correspondances multiples permet de décrire de vastes tableaux binaires, dont les fichiers d'enquêtes socioéconomiques constituent un exemple privilégié: les lignes de ces tableaux sont en général des individus ou observations (il peut en exister plusieurs dizaines de milliers) ; les colonnes sont des modalités de variables nominales, le plus souvent des modalités de réponses à des questions» (Lebart et al., 2006: 187). Les variables continues telles que l'âge, le revenu, l'ancienneté dans le logement et la durée du trajet sont découpées en classes et transformées ainsi en variables nominales. L'outil retenu pour l'analyse typologique est le logiciel SPAD.

23 Construire une typologie, liée au cycle de vie dans notre étude, c'est choisir un groupe homogène de variables actives et adopter un point de vue particulier selon lequel on veut décrire une population. On définit alors, dans une enquête, des groupes homogènes de variables relatives aux différents modules ou thèmes d'une enquête (Lebart et al., 2006: 304).

24 Les variables supplémentaires - passives ou illustratives - ne participent ni à la formation ni à la définition des axes ou des classes, mais elles interviennent a posteriori pour les caractériser. Leur introduction dans l'analyse conforte et enrichit l'interprétation des classes ou des axes définis par les variables actives (Lebart et al., 2006). 
Tableau 2 Description des variables retenues pour l'analyse et détail des modalités associées

\begin{tabular}{|c|c|c|c|c|c|}
\hline $\begin{array}{l}\text { Variables socio- } \\
\text { démographiques }\end{array}$ & $\begin{array}{l}\text { Modalité } \\
\text { (libellé) }\end{array}$ & Descritption & $\begin{array}{l}\text { Indicateurs de } \\
\text { déplacement }\end{array}$ & $\begin{array}{l}\text { Modalité } \\
\text { (libellé) }\end{array}$ & Descritption \\
\hline \multirow{2}{*}{ Sexe } & fem & Femme & \multirow{11}{*}{$\begin{array}{l}\text { Mode de } \\
\text { transport }\end{array}$} & A pé & À pied \\
\hline & masc & Homme & & bicicleta & Vélo \\
\hline \multirow{4}{*}{ Âge } & $-12 \mathrm{ans}$ & & & carro & Voiture particulière \\
\hline & 12-25ans & Classes d'âge & & combinado & $\begin{array}{l}\text { Plusieurs moyens } \\
\text { de transport }\end{array}$ \\
\hline & 25-40ans & & & onibus & Bus public \\
\hline & +60 ans & & & metrô & Métro \\
\hline \multirow{4}{*}{ Activité } & trab & Travailleurs & & moto & Moto \\
\hline & Cours & $\begin{array}{l}\text { Écoliers, collégiens, } \\
\text { lycéens, étudiants }\end{array}$ & & outro meio & $\begin{array}{l}\text { Autre mode } \\
\text { de transport }\end{array}$ \\
\hline & $\mathrm{C} \& \mathrm{~W}$ & Travailleurs étudiant & & táxi & Taxi \\
\hline & & en parallèle & & transporte & Ramassage scolaire ou \\
\hline \multirow{3}{*}{$\begin{array}{l}\text { Ancienneté de } \\
\text { l'individu dans } \\
\text { le logement }\end{array}$} & $\begin{array}{l}\text { Dur-1an } \\
\text { Dur-5ans }\end{array}$ & Nombre d'années & & trem & Train de banlieue \\
\hline & Dur-10ans & $\begin{array}{l}\text { passees dans le } \\
\text { logement enquêté }\end{array}$ & \multirow{5}{*}{$\begin{array}{l}\text { Temps de } \\
\text { trajet vers le } \\
\text { lieu d'activité }\end{array}$} & 0 min & \\
\hline & Dur+10ans & & & $-10 \mathrm{~min}$ & Classes de durée de \\
\hline \multirow{4}{*}{$\begin{array}{l}\text { Niveau } \\
\text { d'éducation }\end{array}$} & $\begin{array}{l}\text { nenhum } \\
\text { fundam }\end{array}$ & $\begin{array}{l}\text { Sans formation } \\
\text { Élémentaire }\end{array}$ & & 20-30min & $\begin{array}{l}\text { trajet vers le lieu } \\
\text { d'activité (école ou } \\
\text { travail) }\end{array}$ \\
\hline & médio & Secondaire & & 30-60min & \\
\hline & superior & Sunérieur & & $+60 \mathrm{~min}$ & \\
\hline & técnico & Technique & \multirow{4}{*}{ Destination } & Centro & Centre \\
\hline \multirow{5}{*}{$\begin{array}{l}\text { Revenu moyen } \\
\text { du ménage }\end{array}$} & Rev_nr & $\begin{array}{l}\text { Revenu non } \\
\text { communiqué }\end{array}$ & & $\begin{array}{l}\text { Anel } 1 \\
\text { Anel } 2\end{array}$ & $\begin{array}{l}\text { Couronne } 1 \\
\text { Couronne } 2\end{array}$ \\
\hline & Rev1 & Moins de 500 reais & & Anel 3 & Couronne 3 \\
\hline & Rev2 & $500-2500$ reais & & Anel 4 & Couronne 4 \\
\hline & Rev3 & $2500-5000$ reais & \multirow{4}{*}{$\begin{array}{l}\text { Distance } \\
\text { parcourue }\end{array}$} & & À proximité (à \\
\hline & Rev4 & Plus de 5000 reais & & DISTR & $\begin{array}{l}\text { l'intérieur du district } \\
\text { de résidence) }\end{array}$ \\
\hline \multirow{4}{*}{$\begin{array}{l}\text { Statut } \\
\text { d'occupation } \\
\text { du logement }\end{array}$} & Alugad & $\begin{array}{l}\text { En location } \\
\text { Logé à titre gratuit }\end{array}$ & & DISTR_adj & $\begin{array}{l}\text { Intermédiaire (vers un } \\
\text { district limitrophe) }\end{array}$ \\
\hline & Ocupad & $\begin{array}{l}\text { (usufruit) } \\
\text { Occupant illégal }\end{array}$ & & DISTR_nadj & $\begin{array}{l}\text { Lointain (vers un } \\
\text { district non limitrophe) }\end{array}$ \\
\hline & Propr_nq & $\begin{array}{l}\text { Propriétaire d'un } \\
\text { logement en cours } \\
\text { de remboursement }\end{array}$ & & & \\
\hline & Propr_q & $\begin{array}{l}\text { Propriétaire d'un } \\
\text { logement remboursé }\end{array}$ & & & \\
\hline
\end{tabular}




\section{Résultats: contribution à la réflexion sur la question des inégalités d'accès aux ressources de la ville}

\section{Accès aux ressources urbaines au regard du lieu de résidence selon l'approche univariée (activité, revenus)}

La figure 2 indique, comme on s'y attendait, que les espaces de mobilité sont beaucoup plus étendus pour le travail que pour les études, et ce, quelle que soit la zone d'enquête. La somme des surfaces elliptiques passe ainsi de $109 \mathrm{~km}^{2}$ pour les études à $772 \mathrm{~km}^{2}$ pour le travail. En d'autres termes, les distances parcourues pour étude sont globalement moins longues, davantage «domocentrées » 25 et les destinations moins variées que pour le travail, ce qui confirme les résultats de l'enquête origine-destination réalisée par la Companhia do metrô en 2007. Ce constat s'explique en partie par une couverture en établissements scolaires globalement régulière sur l'ensemble de l'agglomération et par un aspect fonctionnel dans la mesure où c'est l'école la plus proche qui est généralement fréquentée (logique locale).

Pour la zone d'enquête 09, aucune ellipse n’apparaît, car tous les déplacements effectués au départ de cette zone pour se rendre à un établissement scolaire n'ont qu'une seule destination, le district de résidence. En ce qui concerne l'offre d'emplois, encore fortement concentrée dans le centre et la première couronne (figure 1), elle explique l'orientation centripète des ellipses.

La figure 3 illustre la relation qui existe entre le revenu des ménages et les destinations pour le travail depuis chaque lieu de résidence. Nous dissocions deux classes en regroupant les tranches de revenus initiales présentes dans le questionnaire. Si la somme des surfaces elliptiques des moins aisés $\left(610 \mathrm{~km}^{2}\right)$ est globalement inférieure à celle des plus aisés $\left(842 \mathrm{~km}^{2}\right)$, ce qui tendrait à confirmer les résultats de l'enquête origine-destination de 2007 indiquant que la mobilité augmente avec les revenus, les enquêtes METAL permettent de nuancer localement ce constat.

Sur la figure 3a (moins de 1500 reais), trois zones périphériques $(07,09,10)$ semblent ne pas être polarisées par l'espace central (principal bassin d'emploi de l'agglomération). Leur ellipse n'atteint pas les limites extérieures de l'espace central. Il semble donc que, pour les individus les plus modestes de ces trois zones, l'espace central ne soit pas financièrement accessible, ce qui ne se vérifie pas pour deux autres zones périphériques (04 et 08). Comme en témoignent l'orientation et le net désaxage de leur ellipse par rapport à leur point d'ancrage, les résidants de ces deux zones semblent donc contraints, malgré la distance et leur limite pécuniaire, d'accéder à l'espace central (ou de s'en rapprocher) pour travailler. Ces déplacements sont néanmoins possibles dans la mesure où le réseau ferroviaire de banlieue et le métro (pour la zone 08) passent à proximité (figure 1).

25 Expression proposée par Noël et al. (2001) pour qualifier les espaces d'action des personnes âgées et qui signifie que l'ellipse est centrée sur le lieu de résidence et résume un nombre restreint de lieux visités sur le territoire. Dans notre cas, l'ellipse ne synthétise pas la distribution géographique des différents lieux fréquentés par une même personne, mais bien l'ensemble des lieux visités pour le travail ou les études par l'ensemble des individus ayant participé à l'enquête par zone de résidence. Cette précision étant indiquée, nous nous permettons dans cet article de transposer le sens originel. 
Figure 3 : Dispersion des lieux de destination des individus selon le revenu de leur ménage et leur lieu de résidence (trajets pour le travail)
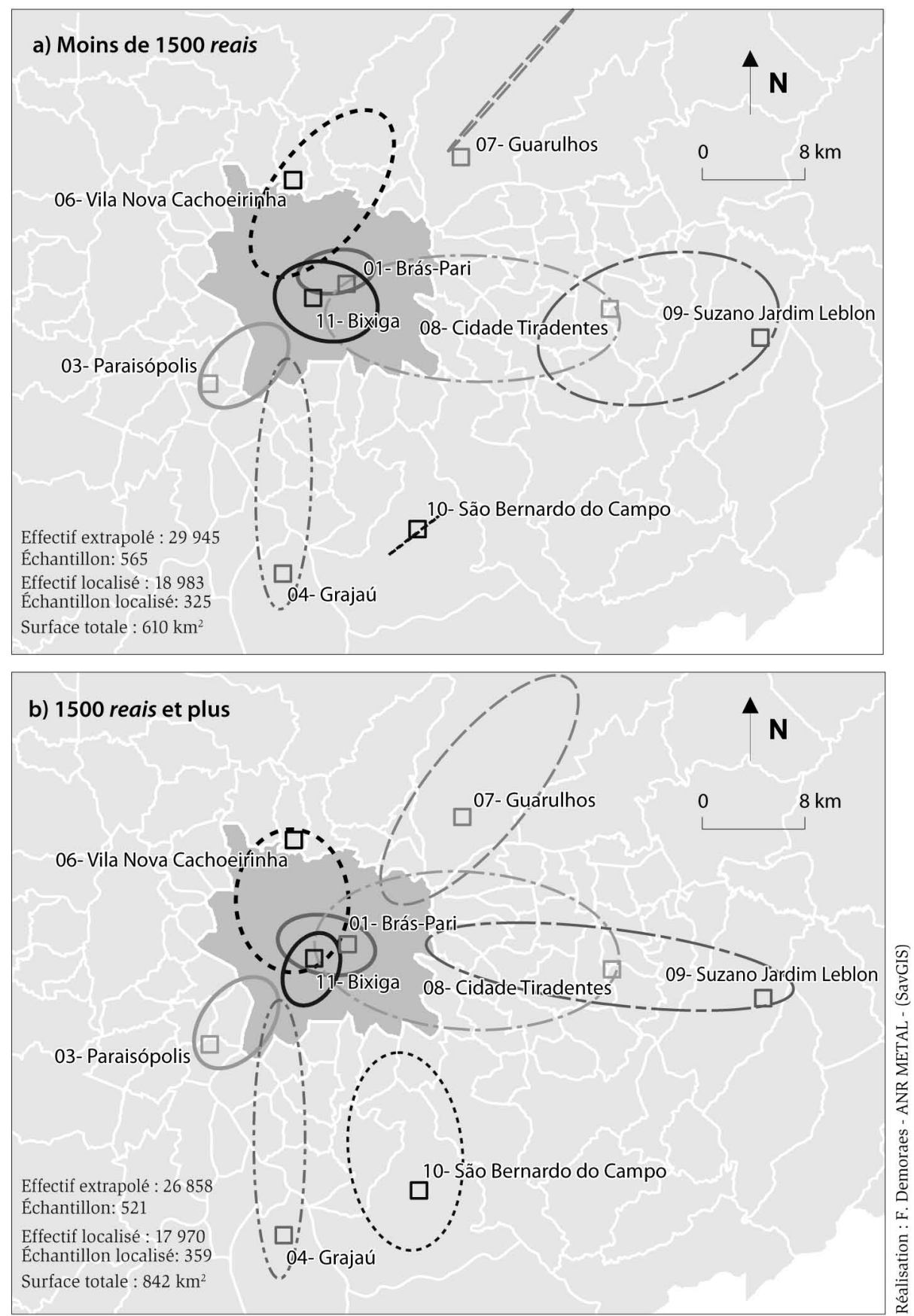

Limite extérieure

de la première couronne

Les divisions à l'extérieur de la première

couronne représentent les districts
Les ellipses représentent un résumé de la dispersion spatiale des lieux pour le travail fréquentés par les individus enquêtés, selon leur lieu de résidence N.B. : le revenu n'est pas renseigné pour 45 actifs 
Sur la figure $3 \mathrm{~b}$ (1500 reais et plus), les espaces de mobilité augmentent fortement (ellipses plus vastes) pour les quatre zones périphériques de l'est (07, 08, 09 et 10). Les ellipses sont toutes nettement orientées vers l'espace central. Pour les zones 03 et 04, la taille, la position et la forme des ellipses ne changent pas beaucoup par rapport à la figure 3a, ce qui montre qu'il n'y a que très peu de possibilités de travail localement quel que soit le niveau de qualification. En ce qui concerne les deux zones à l'intérieur de l'espace central (01 et 11), les ellipses ne varient beaucoup ni en taille, ni en forme par rapport à la figure $3 a$ et restent domocentrées. Cette inertie dans la dispersion des lieux fréquentés pour le travail s'explique localement par la concentration d'une très grande variété d'emplois.

Cette première analyse a permis de différencier les individus suivant deux variables sociodémographiques, prises une à une. Cette première approche, si elle permet d'appréhender certains facteurs qui conditionnent en partie l'inégal accès aux ressources de la ville au regard du lieu de résidence, n'en demeure pas moins partielle. En effet, l'approche univariée proposée ici simplifie les observations, car elle réunit des individus qui se ressemblent sur un critère (même classe de revenus, par exemple) mais qui peuvent être radicalement différents sur d'autres (statut d'occupation du logement, par exemple). Nous proposons donc ci-après d'explorer les espaces de destination déclinés selon une typologie établie à partir d'un plus large ensemble de caractéristiques sociodémographiques individuelles (tableau 2). Comme nous le montrons, cette approche revient à explorer la relation des individus à l'espace à travers le prisme du cycle de vie et de la hiérarchie sociale.

\section{Accès aux ressources urbaines au regard du lieu de résidence suivant l'approche multivariée: déclinaison des ellipses suivant les profils sociodémographiques}

À la lecture du plan factoriel ${ }^{26}$ (figure 4), on retrouve le long du premier axe une distinction entre les individus qui travaillent et ceux qui étudient ou vont à l'école, distinction fortement liée à l'âge, et le long du deuxième axe, une opposition entre propriétaires et locataires dont l'arrivée dans le logement est récente. Le premier axe dissocie aussi les individus à faibles revenus, sans formation, des individus plus aisés ayant suivi une formation supérieure. La position des indicateurs de déplacements (en supplémentaire) autour de l'origine du plan factoriel montre qu'ils sont peu illustratifs de la structure sociodémographique des individus, en dehors de l'usage de certains modes de transport. Ainsi, la voiture, davantage associée aux travailleurs plutôt aisés, et le transport scolaire, logiquement associé aux individus jeunes aux études, s'opposent nettement sur l'axe 1. Une autre opposition s'observe sur l'axe 2 entre les déplacements en métro, plutôt associés aux propriétaires, et les déplacements en vélo, rattachés aux locataires récemment installés. Enfin, la position du nom des zones d'enquête (en supplémentaire) autour de l'origine du plan factoriel renseigne également sur leur faible pouvoir discriminant. Ceci s'explique par le choix des zones d’enquête caractérisées par une grande hétérogénéité sociodémographique, sauf à Bixiga (tableau 1).

26 Nous rappelons que les taux d'inertie relatifs aux axes factoriels sont dépendants du codage préliminaire de l'information brute; dans le cadre d'une analyse des correspondances multiples, ils dépendent par conséquent du codage disjonctif complet et sont généralement faibles. Il faut donc éviter de les interpréter en termes «d'information», car ils rendent compte de mesures pessimistes de l'information extraite à partir de l'axe (Lebart et al., 2006: 205 et 223). 


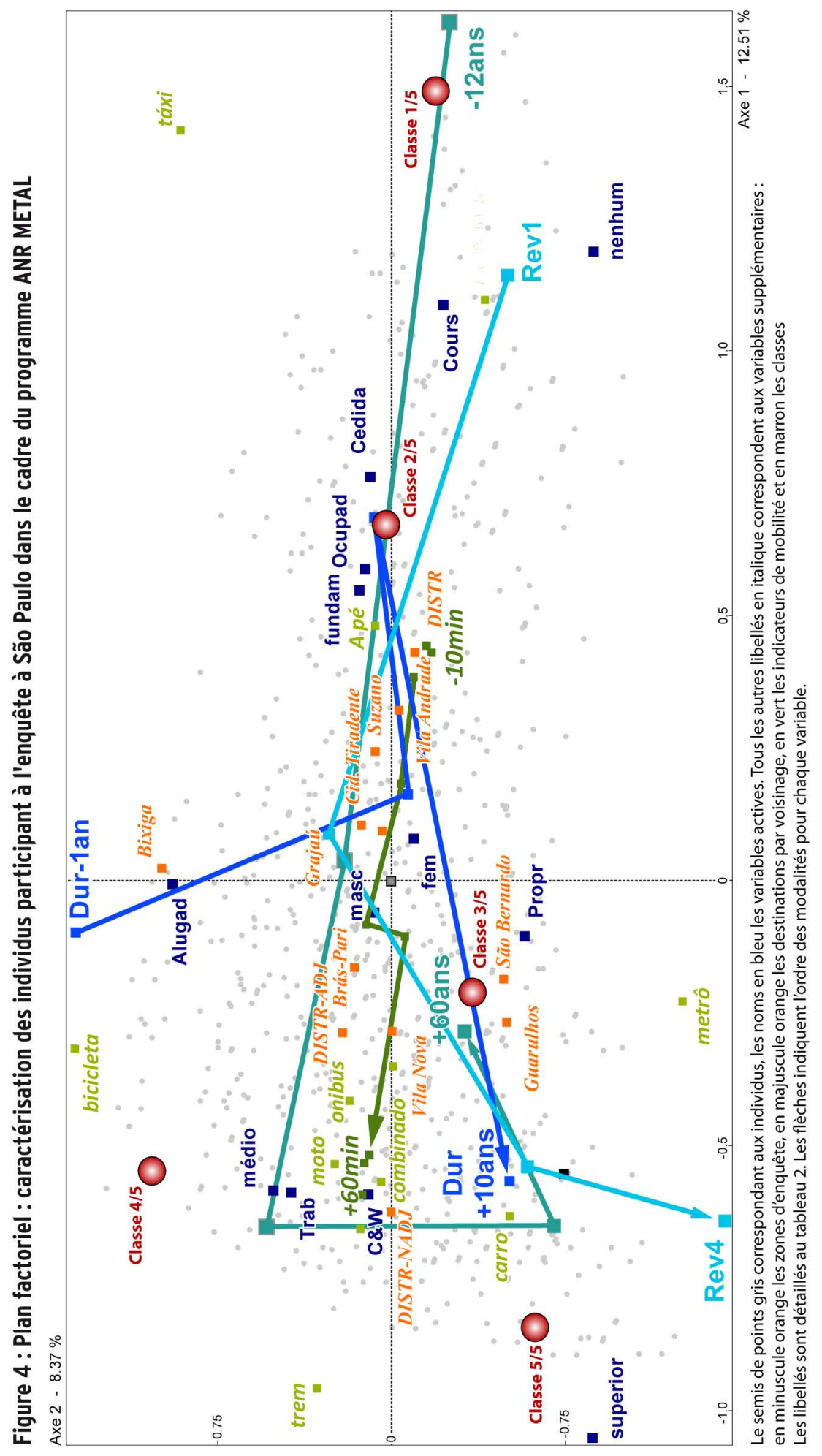


La classification ascendante hiérarchique permet de dégager cinq classes d’individus décrites à la figure 5 par les modalités les plus caractéristiques. Ces classes sont projetées en éléments supplémentaires sur le plan factoriel principal (figure 4).

- La classe 1/5 (19\% de l'échantillon extrapolé) regroupe les individus allant à l'école. Ceux-ci sont jeunes (moins de 12 ans), étudient à proximité de leur lieu de résidence (dans le même district) et se rendent à leur établissement scolaire principalement à pied. Ce sont les "élèves».

- La classe 2/5 (10\% de l'échantillon extrapolé) rassemble des individus défavorisés, logés à titre gratuit, ayant des revenus modestes, se déplaçant principalement à pied, à proximité de leur lieu de résidence (dans le même district). Ce sont les «démunis».

- La classe 3/5 (22\% de l'échantillon extrapolé) réunit de jeunes actifs étudiant en parallèle et des individus aux études. Eux se déplacent à proximité de leur lieu de résidence (dans le même district), principalement à pied ou en bus. Ce sont les «étudiants».

- La classe 4/5 (25\% de l'échantillon extrapolé) englobe les travailleurs, majoritairement âgés de 25 à 40 ans, ayant suivi des études secondaires, se déplaçant principalement en bus. On note également dans cette classe une forte proportion de locataires. Ce sont les «actifs de classe moyenne».

- La classe 5/5 (24\% de l'échantillon extrapolé) regroupe des travailleurs, majoritairement âgés de 40 à 60 ans, se déplaçant en voiture, ayant un niveau d'études supérieures et parcourant des distances assez longues (vers des districts non limitrophes de leur lieu de résidence). Ce sont les «actifs aisés plus âgés».

Les classes reflètent des différenciations marquées par le cycle de vie (âge, activité) et par la hiérarchie sociale (revenu, niveau d'étude). Le choix de retenir cinq classes correspond à un compromis permettant de disposer de classes d'effectifs suffisants pour le calcul des ellipses et qui structurent de façon significative la population étudiée. Ces classes montrent, à ce niveau de regroupement et par conséquent de façon schématique, que plus on avance en âge, plus les revenus augmentent, et que cette covariation est également fonction du niveau d'étude pour la population étudiée.

La figure 6 représente une ellipse pour chacune des cinq classes sociodémographiques définies précédemment, par lieu de résidence. Premier constat, les espaces de mobilité quotidienne reflètent une forte hétérogénéité suivant le lieu de résidence; la position, la forme, l'orientation et la taille des ellipses varient beaucoup en fonction de la zone étudiée. Deuxième constat, à de rares exceptions près, les individus d'une même classe d'appartenance n'accèdent pas de la même manière aux ressources urbaines (étude/ travail) suivant leur lieu de résidence. Troisième constat, les ellipses de chaque classe sont tantôt assez semblables entre elles pour une même zone d'enquête, tantôt très contrastées. Ainsi, trois catégories de lieu de résidence se dégagent, compte tenu de la relation qu'entretiennent les individus à l'espace: 
- Pour les zones d'enquête du centre (01 - 11) et du centre-ouest (03), les ellipses ne sont pas très différenciées d'une classe à l'autre et sont plutôt ramassées. En somme, à proximité de ces trois lieux de résidence, la ville offre emplois et lieux d'étude pour tous les individus quelle que soit leur classe sociodémographique d’appartenance.

- Pour les trois zones d'enquête du nord (06 - 07) et du sud-est (10), les ellipses sont un peu plus étirées, sauf pour les classes 1 et 2 qui n'apparaissent pas toujours, soit du fait d'un nombre de destinations inférieur à deux, soit du fait d'un trop faible effectif (inférieur à 5). Pour la classe $1 / 5$ (scolaires), sans ellipse pour les zones 07 et 10, cette observation confirme le fait que les trajets pour étude se font à proximité du lieu de résidence $^{27}$. Pour la classe $2 / 5$ (individus modestes), sans ellipse pour la zone 07 , cela corrobore les caractéristiques de cette classe (déplacements à pied, à proximité du lieu de résidence). On observe ainsi une polarisation des flux localement (en particulier pour les classes $1 / 5$ et $2 / 5$ ) qui s'explique à la fois par la taille des districts localement, le niveau de richesse modeste des individus de ces deux classes, limitant le recours à un mode de transport motorisé, et par la présence de centralités secondaires à proximité ${ }^{28}$, notamment au niveau de l'aéroport et dans le secteur $\mathrm{ABC}$. Cette polarisation confirme les résultats obtenus à partir de l'enquête origine-destination de 2002 (Aranha, 2005). On observe en parallèle, pour ces trois zones, une relation de dépendance assez forte vis-à-vis du centre et des deux premières couronnes (compte tenu de l'orientation des ellipses) pour les classes 3/5 (étudiants), 4/5 et 5/5 (travailleurs).

- Pour les trois zones d'enquête les plus périphériques (04-08 - 09), les ellipses sont comparativement plus grandes et plus allongées (déplacements plus longs dans un faisceau de directions assez restreint), quelle que soit la classe (sauf là encore pour la 1/5). Les ellipses sont par ailleurs orientées pour la plupart vers le centre. Cette configuration s'explique à la fois par la localisation de ces trois zones d'enquête par rapport à l'ensemble de l'agglomération (sur les marges) et par un manque d'emplois locaux qui oblige les actifs à se déplacer loin pour accéder à du travail. Dans les zones 04 et 09, les actifs âgés les plus aisés, se déplaçant en voiture et ayant un niveau d'étude supérieur (classe 5/5), semblent pouvoir accéder à toute l'agglomération (et pas seulement au centre et à la première couronne), dans la mesure où sont associées à ces individus les ellipses qui sont à la fois les plus grandes et les moins aplaties (destinations plus variées). C'est également dans ces trois zones qu'on observe les contrastes les plus marqués entre les classes.

27 Nous rappelons ici que l'offre éducative est plus équitablement répartie dans l'ensemble de l'agglomération que l'offre d'emploi (tout au moins pour le primaire et le secondaire).

28 La consolidation de centralités secondaires en périphérie des agglomérations attire un nombre sans cesse grandissant de navettes. Paquette montre notamment, à Mexico, que «l'existence de ressources locales contribue par ailleurs à limiter les besoins - des citadins - en matière de déplacement pour réaliser un certain nombre d'activités quotidiennes. À l'heure où l'on valorise de plus en plus la mobilité $[. .$. ] et où, inversement, les formes de sédentarité ont tendance à être interprétées comme des indices de relégation, voire de pauvreté, le centrage sur les espaces locaux des périurbains modestes de Mexico ne doit pas être lu comme une assignation à résidence, mais au contraire comme une forme d'ancrage qui permet le bon déroulement de la vie quotidienne, ainsi que le fonctionnement de la métropole» (2010: 172). 


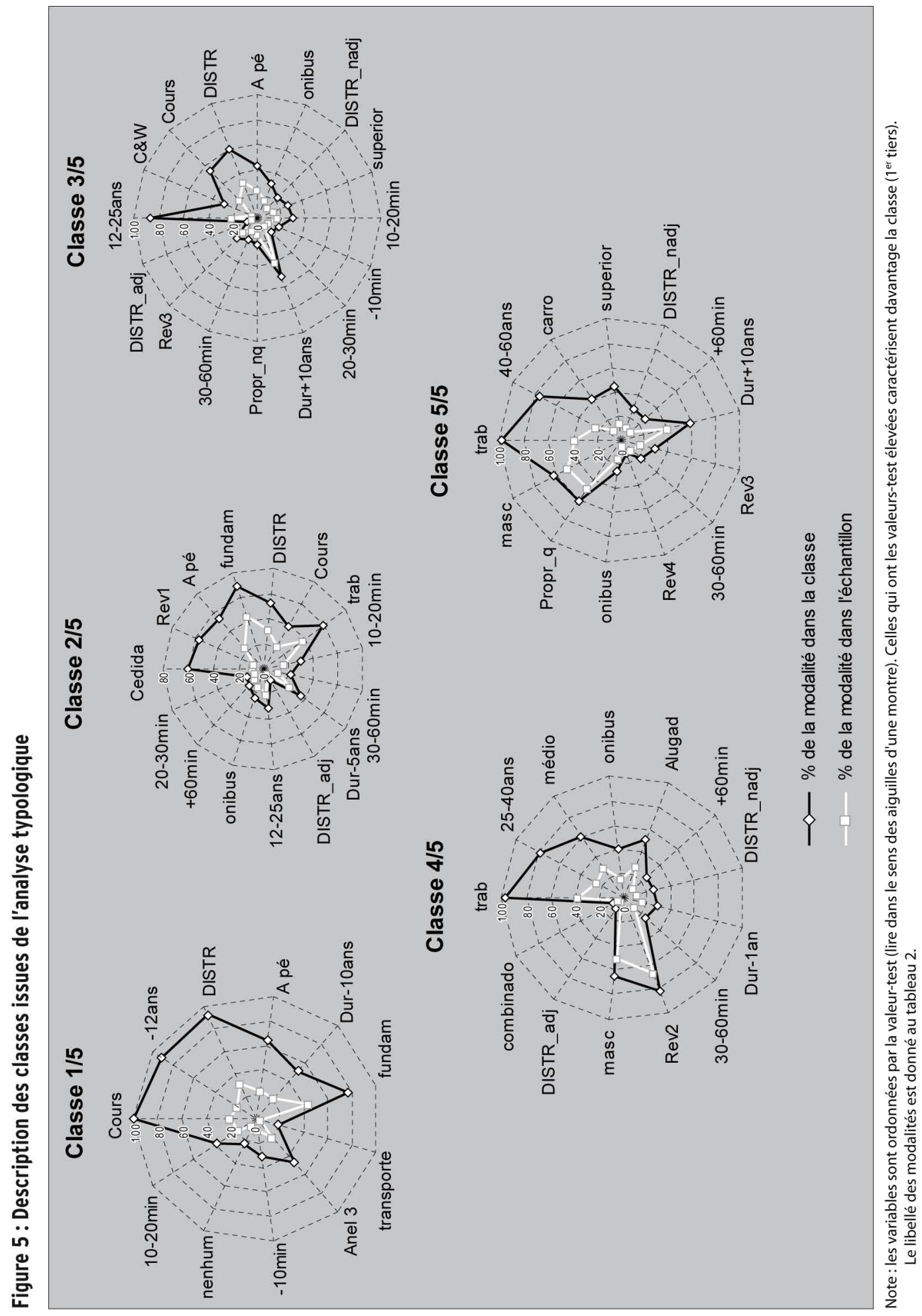




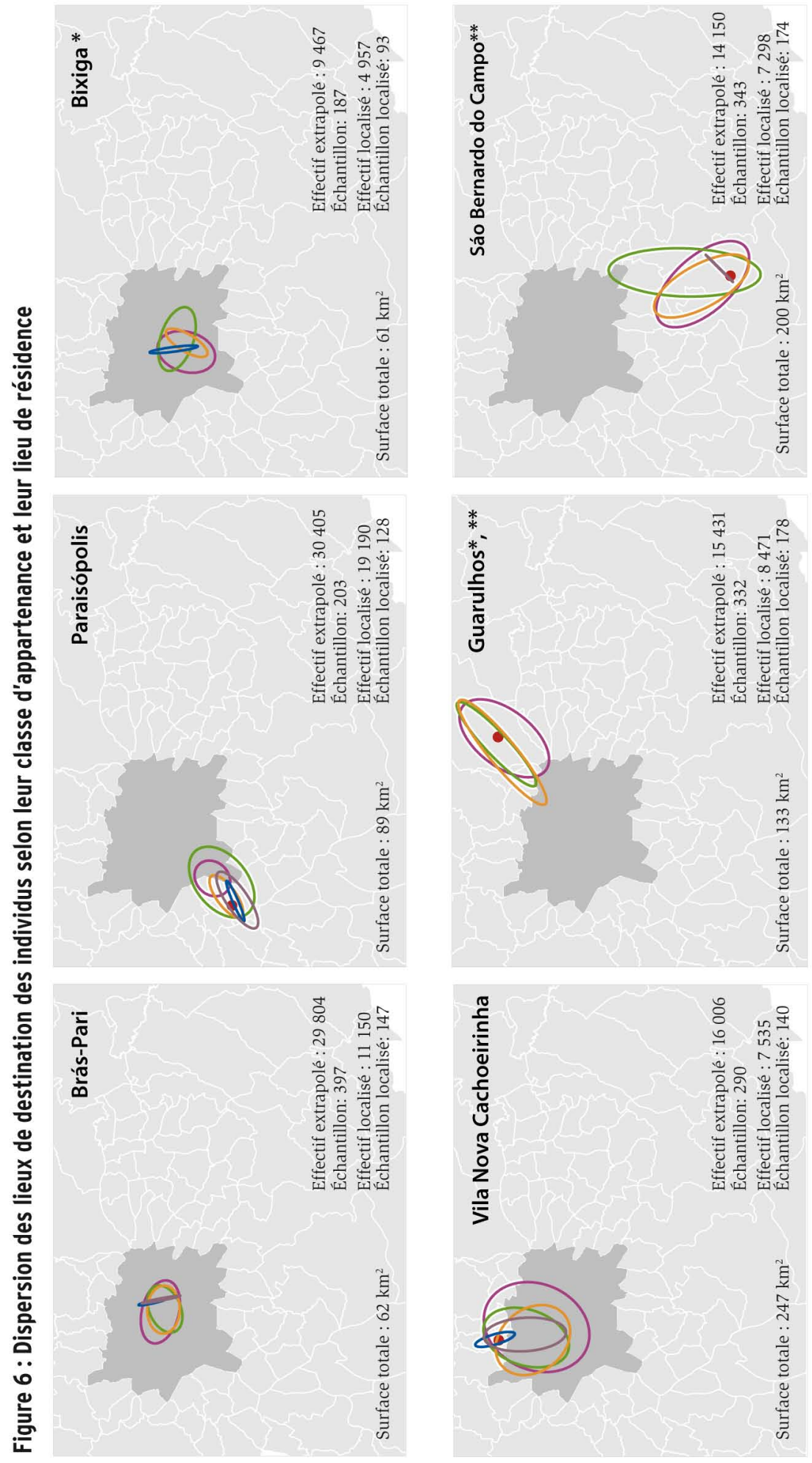



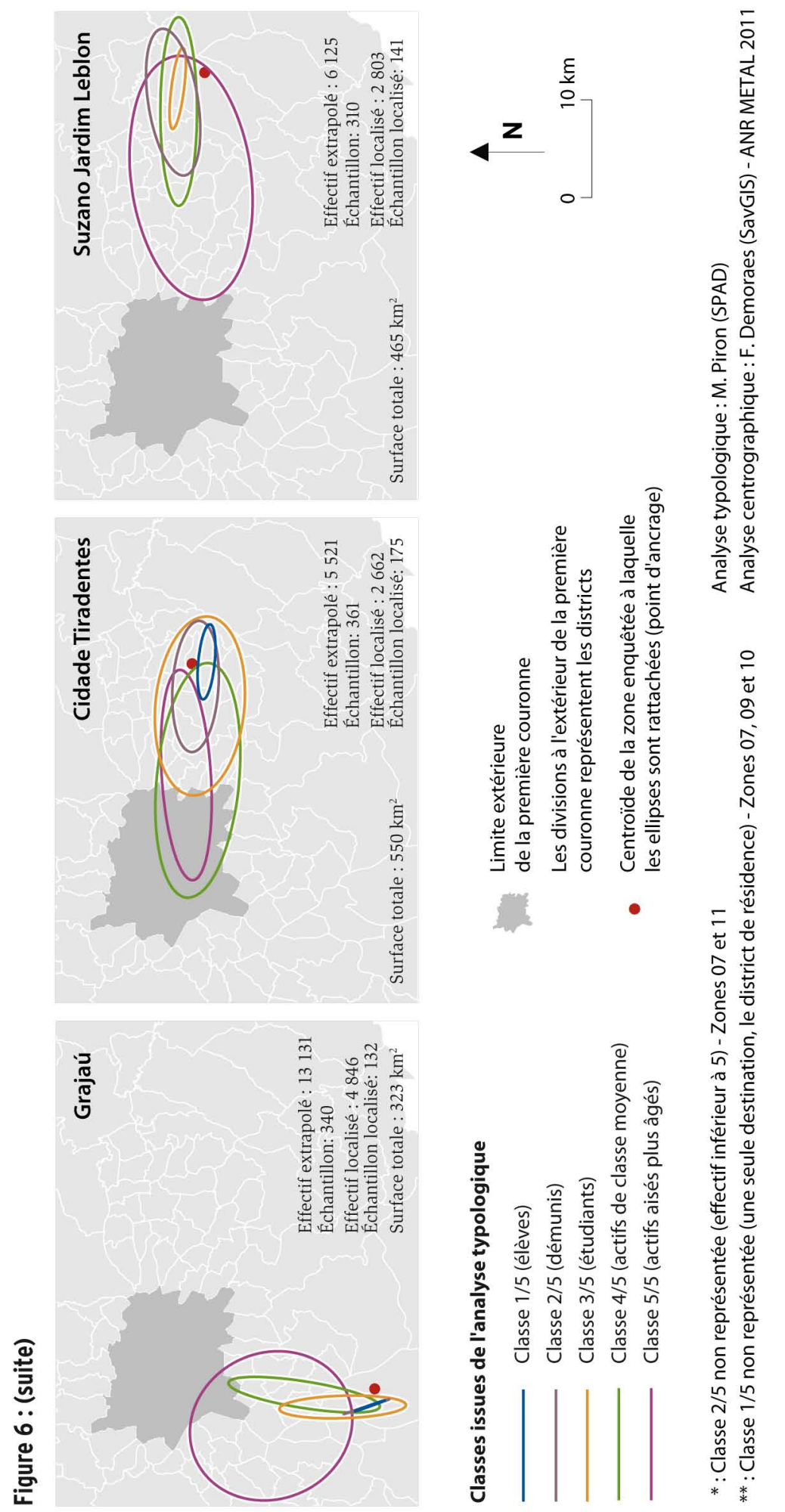
Cet examen visuel confirme finalement que l'accès aux ressources urbaines (études/ travail) est fortement conditionné par le lieu de résidence et, dans une moindre mesure, par le cycle de vie et la hiérarchie sociale. Par ailleurs, un gradient se dessine à l'échelle de l'agglomération de São Paulo : les inégalités d'accès se creusent avec l'éloignement au centre alors que l'espace central offre aux individus toutes les ressources, indépendamment de leur profil sociodémographique. L'orientation centripète des espaces de mobilité quotidienne pour les études et le travail confirme la persistance de la forte attractivité de l'espace central, même si, au-delà d'une certaine distance, les classes les plus modestes de la périphérie n’y ont plus accès.

Dans le même ordre d’idées, ces analyses nous interpellent aussi sur le «découplage spatial» entre lieu de résidence et lieu d'activité, qui s'accentue au fur et à mesure qu’on gagne la périphérie. Ce phénomène s’observe au vu du désaxage des ellipses par rapport à leur point d'ancrage (zone d'enquête). Notons que, contrairement à la notion de spatial mismatch introduite par Kain en 1968 pour décrire la dissociation spatiale très nette qui existait à Chicago et à Détroit entre les bassins d'emploi et les lieux de résidence des populations en particulier noire-américaine à bas revenus ${ }^{29}$, le découplage spatial le plus marqué concerne ici avant tout les actifs aisés de la périphérie. Cette observation renvoie en partie à la stratégie résidentielle de ces individus qui ancrent leur domicile loin du centre, dans des complexes résidentiels (immeubles et pavillonnaires) fermés, plus sécurisés et plus homogènes socialement (entre soi, milieu affinitaire), un choix résidentiel qui implique en contrepartie de longues navettes quotidiennes en automobile.

\section{Conclusion}

La démarche, en partie exploratoire, élaborée dans cet article sur des données d'enquête a permis d'apporter des éclairages sur les disparités qui existent à São Paulo pour accéder à la ville. Cette démarche repose sur l'usage combiné de deux méthodes éprouvées, simples à mettre en œuvre (l'analyse centrographique et l'analyse typologique) même si leur application à des recueils issus d'enquête soulève quelques difficultés qui renvoient à la qualité des données en entrée (précision de la localisation, question des effectifs, nombre minimal de destinations). Dans notre cas, ces méthodes ont permis de synthétiser et visualiser les espaces de mobilité quotidienne et de les mettre en balance au regard du lieu de résidence, de la position des individus dans leur cycle de vie et de la hiérarchie sociale. Ces premiers résultats constituent un apport aux essais de décryptage des articulations entre les différents registres de la mobilité (notamment quotidienne et résidentielle), objet d'un nombre grandissant de travaux, tant les enjeux associés sont nombreux, en particulier dans le domaine de la gestion urbaine. Dans le prolongement de cet article, l'exploitation des entretiens approfondis nous permettra d'enrichir ces premiers résultats exploratoires et de mieux comprendre les disparités qu'on observe parmi les lieux fréquentés au quotidien. Nous pourrons notamment savoir si les espaces d'action reflètent des mobilités contraintes, des mobilités choisies, des mobilités contraignantes, autant d'indicateurs pour appréhender plus finement les inégalités sociospatiales d'accès à la ville.

29 Le thème du spatial mismatch a été largement développé dans les études sur la ségrégation au cours de ces 40 dernières années. Un intéressant essai pour en modéliser les mécanismes d'un point de vue théorique est proposé par Gobillon et al. (2007). 
À São Paulo, comme dans de nombreuses autres agglomérations en Amérique latine, le découplage spatial entre le lieu de résidence et les bassins d'emploi s'observe avant tout dans les espaces périphériques. Ce constat renvoie à un modèle de développement urbain extensif peu dense, caractérisé par une spécialisation fonctionnelle, où les emplois se concentrent majoritairement dans l'espace central, et qui est structuré par un réseau viaire à grande capacité rendant possible la circulation à grande vitesse et le parcours de longues distances. Dans ce modèle, la place des transports motorisés est centrale et le schéma conceptuel d'équité appliquée à l'accessibilité, mis à mal. Le découplage spatial met également en question la notion de développement durable. Quel est le degré de découplage spatial maximal acceptable dans les villes de demain? La consolidation en périphérie de centralités secondaires sera-t-elle suffisante pour polariser des navettes en nombre sans cesse grandissant? Est-ce une solution viable face à l'hypermobilité ou, au contraire, une telle consolidation ne risque-t-elle pas d'accentuer la fragmentation des grandes agglomérations? 


\section{Bibliographie}

ÂNTICO, Cláudia (2005) Deslocamentos pendulares na região metropolitana de São Paulo. São Paulo Perspectivas, vol. 19, $\mathrm{n}^{\circ} 4$, p. 110-120. [En ligne]. http://www.scielo. br/pdf/spp/v19n4/v19n4a07.pdf

ARANHA, Valmir (2005) Mobilidade pendular na metrópole paulista. São Paulo Perspectivas, vol. 19, n4 ${ }^{\circ}$, 96-109. [En ligne]. http://www.scielo.br/pdf/spp/v19n4/ v19n4a06.pdf

BACCAÏNI, Brigitte (1996) Les trajets domicile-travail en Île-de-France. Contraste entre catégories socioprofessionnelles. Économie et Statistique, n²94-295, p. 109-126.

BACHI, Roberto (1963) Standard distance measure and related methods for spatial analysis. Papers in Regional Sciences, vol. 10, ${ }^{\circ} 1$, p. 73-132.

BERGER, Martine (2004) Les périurbains de Paris. Paris, CNRS.

BERGER, Martine et BEAUCIRE, Francis (2002) Mobilité résidentielle et navette. Les arbitrages des ménages d'Île-deFrance. Dans Jean-Pierre Lévy et Françoise Dureau (dir.) L'accès à la ville. Les mobilités spatiales en questions. Paris, L'Harmattan, p. 141-166.

BORSDORF, Axel (2003) Hacia la ciudad fragmentada. Tempranas estructuras segregadas en la ciudad latinoamericana. Scripta Nova. Revista electrónica de geografía y ciencias sociales. Universidad de Barcelona, vol. VII, n 146 (122). [En ligne]. http://www.ub.edu/geocrit/sn/ sn-146\%28122\%29.htm

CLICHEVSKY, Nora (2000) Informalidad y segregación urbana en América Latina. Una aproximación. Serie Medio Ambiente y Desarrollo, $\mathrm{n}^{\circ} 28$. [En ligne]. http://www. eclac.org/publicaciones/xml/8/5638/ lcl1430e.pdf

CIA. DO METRO (2008) Pesquisa Origem e Destino 2007. Região Metropolitana de São Paulo. Síntese das informações - pesquisa domiciliar. São Paulo: STM/Cia. Do Metrô.
DELAUNAY, Daniel (2010) Mobilités, ségrégations résidentielles et bonus démographique dans la zone métropolitaine de Santiago du Chili. Dans Monique Bertrand (dir.) Mobilité, pauvretés: les villes interrogées. Revue Tiers Monde, n²01, p. 65-85.

DEMORAES, Florent, GOUËSET, Vincent, PIRON, Marie, FIGUEROA, Oscar et ZIONI, Silvana (2010) Mobilités quotidiennes et inégalités socio-territoriales à Bogotá, Santiago du Chili et São Paulo. Espace, Populations, Sociétés, n²010-2 "Nouvelles mobilités dans les Suds», p. 349-364.

DONALD, Janelle et GOODCHILD, Michael (1983) Diurnal patterns of social group distribution in Canadian cities. Economic Geography, vol. 59, n² 4, p. 403-425.

DUREAU, Françoise (2006) Habiter la ville: stratégies et mobilités résidentielles. Dans Françoise Dureau, Vincent Gouëset et Evelyne Mesclier (dir.) Géographies de l'Amérique latine. Rennes, Presses universitaires de Rennes, p. 263-292.

DUREAU, Françoise, GOUËSET, Vincent, LE ROUX, Guillaume et LULLE, Thierry (2012) Cambios urbanos y evolución de las desigualdades en el acceso a los recursos de la metrópoli. Un estudio de caso en unos barrios del occidente de Bogotá, Communication présentée au X seminario ACIUR (Asociación Colombiana de Investigadores Urbano regionales), septiembre 2012, Universidad Javeriana, Bogota. [En ligne]. http://halshs.archivesouvertes.fr/docs/00/74/51/58/PDF/ Dureau_Goueset_LeRoux_Lulle_2012_ ACIUR_Calle_80_com.pdf

DUREAU, Françoise, BARBARY, Olivier, MICHEL, Alain et LORTIC, Bernard (1989) Sondages aréolaires sur image satellite pour des enquêtes socio-démographiques en milieu urbain. Paris, Orstom. [En ligne]. http://horizon.documentation.ird.fr/exl-doc/pleins_textes/ pleins_textes_7/divers2/30031.pdf 
FIGUEROA, Oscar (2005) Transporte urbano y globalización. Políticas y efectos en América latina. Revista Eure, vol. XXXI, n 94 , p. 41-53. [En ligne]. http://www.scielo.cl/ scielo.php?script=sci_arttext\&pid=S0250$71612005009400003 \& \operatorname{lng}=$ en\&nrm=iso\&ig nore $=$. html

GOBILLON, Laurent, SELOD, Harris et ZENOU, Yves (2007) The mechanisms of spatial mismatch. Urban Studies, vol.44, $n^{\circ} 12$, p. 2401-2427.

IMBERT, Christophe, DUREAU, Françoise et GIROUD, Mathieu (2009) Méthodes d'analyse des mobilités urbaines des ménages: réflexions autour de l'enquête «Déplacements Poitiers». Dans Janica Macchi (dir.) Geografie del popolamento, Casi di studio, metodi e teorie. Atti della giornata di studi Grosseto, 24-26 settembre 2008, Università degli Studi di Siena, p. 89-96.

JANOSCHKA, Michael (2002) El nuevo modelo de la ciudad latinoamericana: fragmentación y privatización. Revista Eure, vol. 28, n ${ }^{\circ}$, p. 11-20. [En ligne]. http://www.scielo.cl/scielo.php?script $=$ sci_arttext\&pid $=$ S0250$71612002008500002 \& \operatorname{lng}=e s \& n r m=i s o \&$ tlng=es

KAIN, John F. (1968) Housing segregation, Negro employment and metropolitan decentralization. Quarterly Journal of Economics, vol. 82, n² 2, p. 175-197.

KILROY, Austin (2007) Intra-urban spatial inequality: Cities as "urban regions”, Reshaping Economic Geography, World Development Report 2009. [En ligne]. http://siteresources. worldbank.org/INTWDR2009/Resources/4231006-1204741572978/Kilroy2. pdf

LEBART, Ludovic, PIRON, Marie et MORINEAU, Alain (2006) Statistique exploratoire multidimensionnelle: visualisation et inférence en fouille de données. Dunod, Paris.
LÉVY, Jean-Pierre (2009) Mobilité urbaine: des pratiques sociales aux évolutions territoriales. Dans Françoise Dureau et Marie-Antoinette Hily (dir.) Les mondes de la mobilité. Rennes, Presses universitaires de Rennes, p. 107-136.

LÉVY, Jean-Pierre et DUREAU, Françoise (dir.) (2002) L'accès à la ville. Les mobilités spatiales en question. Paris, L'Harmattan.

LORD, Sébastien, JOERIN, Florent et THÉRIAULT, Marius (2009) Évolution des pratiques de mobilité dans la vieillesse: un suivi longitudinal auprès d'un groupe de banlieusards âgés. Cybergeo: revue européenne de géographie, article $\mathrm{n}^{\circ} 444$, [En ligne]. http://cybergeo.revues. org/22090

LUNGO, Mario et BAIRES, Sonia (2001) Sociospatial segregation and urban land regulation in Latin American cities, proceeding of the International Seminar on Segregation in the City held from July 26-28, 2001, at the Lincoln Institute. [En ligne]. http:// citeseerx.ist.psu.edu/viewdoc/download? doi=10.1.1.200.8130\&rep=rep1\&type=pdf

MONTEZUMA, Ricardo (2003) Ciudad y transporte. La movilidad urbana. Dans Marcello Balbo, Ricardo Jordan et Daniela Simioni (dir.) La ciudad inclusiva, Santiago du Chili. Cuadernos de la CEPAL, ${ }^{\circ} 88$, p. 175-191. [En ligne]. http://www.eclac.org/ publicaciones/xml/7/14237/lcg2210p.pdf

MORENCY, Catherine (2006) Étude de méthodes d'analyse spatiale et illustration à l'aide de microdonnées urbaines de la Grande Région de Montréal. Les Cahiers scientifiques du transport, ${ }^{\circ} 49$, p. 77-102. [En ligne]. http://afitl.ish-lyon.cnrs.fr/tl_ files/documents/CST/N49/Moren49.pdf

NOËL Nathalie, VILLENEUVE, Paul Y. et LEE-GOSSELIN, Martin (2001) Aménagement du territoire et espaces d'action. Identification des déterminants des stratégies de déplacements de cyclistes de la région de Québec à l'aide d'un SIG. Revue internationale de géomatique, vol.11/3-4, p. 381-404. 
PAQUETTE, Catherine (2010) Mobilité quotidienne et accès à la ville des ménages périurbains dans l'agglomération de Mexico. Une lecture des liens entre pauvreté et mobilité. Revue Tiers Monde, $n^{\circ} 201$, p. 157-175.

POCHET, Pascal et ROUTHIER, Jean-Louis (2002) Mobilité résidentielle et allongement des distances domicile-travail dans la région urbaine de Lyon. Dans Françoise Dureau et Jean-Pierre Lévy (dir.) L'accès à la ville. Les mobilités spatiales en questions. Paris, L'Harmattan, p. 167-184.

RODRIGUEZ, Jorge (2007) Segregación residencial, migración y movilidad especial. El caso de Santiago de Chile. Cadernos metrópole, $\mathrm{n}^{\circ} 17$, p. 135-168.

RODRIGUEZ, Jorge et ARRIAGADA, Camilo (2004) Segregación residencial en la Ciudad Latinoamericana. Revista Eure, $\mathrm{n}^{\circ} 30$, p. 5-24. [En ligne]. http://www.scielo.cl/ scielo.php?script=sci_arttext\&pid=S0250$71612004008900001 \& \operatorname{lng}=$ en\&nrm=iso\&ig nore $=$.html

SOURIS, Marc, DEMORAES, Florent, SERRANO, Tania et HABERT, Elisabeth (2007), Manuels de référence du logiciel SavGIS. [En ligne]. http://www.savgis.org/SavGIS/documentation/manuels.html

THÉRIAULT, Marius (1994) MapStat-Module d'analyse spatiale fonctionnant sous MapInfo. Québec, Centre de recherche en aménagement et développement - CRAD, Université Laval.

VASCONCELLOS, Eduardo (1996) Transporte urbano nos países em desenvolvimento. São Paulo, Ed. Annablume.

VASCONCELLOS, Eduardo (2005) Transport metabolism, social diversity and equity: The case of São Paulo, Brazil. Journal of Transport Geography, vol. 13, n 4 , p. 329-339.

VILLAÇA, Flávio (2007) Espaço intra-urbano no Brasil. São Paulo: Studio Nobel, FAPESP, Lincoln Institute.
VILLAÇA, Flávio et ZIONI, Silvana (2005) Os transportes sobre trilhos na região metropolitana de São Paulo: o poder público acentuando a desigualdade. Rio de Janeiro: CBTU. [En ligne]. http://www. cbtu.gov.br/monografia/seminario/sp/ flavioesilvania.pdf 